\title{
The 2D continuum radiative transfer problem
}

\section{Benchmark results for disk configurations}

\author{
I. Pascucci ${ }^{1}$, S. Wolf ${ }^{1,2}$, J. Steinacker ${ }^{1,3}$, C. P. Dullemond ${ }^{4}$, Th. Henning ${ }^{1}$, G. Niccolini ${ }^{5}$, P. Woitke ${ }^{6}$, and B. Lopez ${ }^{5}$ \\ 1 Max-Planck-Institut für Astronomie, Königstuhl 17, 69117 Heidelberg, Germany \\ 2 California Institute of Technology, 1201 East California Blvd, Mail Code 105-24, Pasadena, CA 91125, USA \\ 3 Astrophysikalisches Institut und Universitäts-Sternwarte, Schillergässchen 2-3, 07745 Jena, Germany \\ 4 Max-Planck-Institut für Astrophysik, Karl-Schwarzschild-Str. 1, 85741 Garching, Germany \\ 5 Observatoire de la Côte d'Azur, Département Fresnel, UMR 6528, BP 4229, 06034 Nice Cedex 4, France \\ 6 Zentrum für Astronomie und Astrophysik, TU Berlin, Hardenbergstraße 36, 10623 Berlin, Germany
}

Received 26 July 2002 / Accepted 2 January 2004

\begin{abstract}
We present benchmark problems and solutions for the continuum radiative transfer (RT) in a 2D disk configuration. The reliability of three Monte-Carlo and two grid-based codes is tested by comparing their results for a set of well-defined cases which differ for optical depth and viewing angle. For all the configurations, the overall shape of the resulting temperature and spectral energy distribution is well reproduced. The solutions we provide can be used for the verification of other RT codes. We also point out the advantages and disadvantages of the various numerical techniques applied to solve the RT problem.
\end{abstract}

Key words. radiative transfer - stars: circumstellar matter - methods: numerical

\section{Introduction}

Observations show that many astrophysical sources such as young stellar objects (YSOs), post-AGB stars and active galactic nuclei (AGN) are surrounded by dust. Dust grains scatter, absorb and re-emit radiation originating from the primary energy sources, thus modifying their spectral energy distributions (SEDs). Moreover many embedded objects cannot be directly studied in the visible, since dust may entirely obscure them at optical wavelengths. Their structure can be only inferred from the thermal dust emission. Therefore, modelling of their intensity and polarization maps as well as their SEDs is necessary. This can only be done by solving the radiative transfer (RT) equation (e.g. Yorke 1985). Analytical solutions for this equation do exist only for the simplest cases, far from representing the complexity of dust-enshrouded objects. Hence, the development of sophisticated numerical RT codes is unavoidable.

Early attempts for spherically symmetric configurations were performed by Hummer \& Rybicki (1971), Scoville \& Kwan (1976) and Leung (1976) including rough assumptions such as grey opacity and/or neglecting scattering. The first formal solution for the dust continuum in spherical geometry was obtained by Rowan-Robinson (1980), directly integrating the RT equation, an operation known as ray-tracing.

Send offprint requests to: I. Pascucci, e-mail: pascucci@mpia-hd.mpg.de
Since then many other codes treating 1-D slab or spherical configurations (e.g. Yorke \& Shustov 1981; Lefèvre et al. 1982; Martin et al. 1984; Rogers \& Martin 1984; Henning 1985; Groenewegen 1993; Winters et al. 1994) or the inverse RT problem (Steinacker et al. 2002b) have been developed. Nevertheless, it became soon clear that a 1-D geometry is often too restrictive. Distinct non-spherical features such as bipolar outflows (e.g. Bachiller 1996), bipolar reflection nebulae (e.g. Lenzen 1987) and disks (e.g. McCaughrean \& O’Dell 1996) are typical of many astronomical objects. Nowadays, multidimensional codes implementing different methods and numerical schemes are being applied to treat the RT in such configurations.

In contrast to hydrodynamical simulations, benchmark tests for radiative transfer computations are rare. The only practical approach to test the reliability of RT calculations is to compare solutions of well-defined problems by several independent codes. This has been done for the 1D case by Ivezic et al. (1997). A benchmark project for 1-D plane-parallel RT and vertical structure calculations for irradiated passive disks is available on the web $^{1}$. As for 1-D RT in molecular lines, a comparison of results from different codes has been performed by van Zadelhoff et al. (2002) ${ }^{2}$. Going from spherical symmetry to $2 \mathrm{D}$ and $3 \mathrm{D}$ spatial configurations, we add two or three more

\footnotetext{
${ }^{1}$ http://www.mpa-garching.mpg.de/PUBLICATIONS/DATA/ radtrans/benchmarks/

2 see also: http://www.strw.leidenuniv.nl/ radtrans/
} 
variables to the RT problem. Numerically, this implies $10^{4}$ or $10^{6}$ more numbers to store when a decent resolution of 100 points in each variable is used. In addition, the geometry makes the solution of the integro-differential RT equation more complex. This explains why benchmark tests for $2 \mathrm{D}$ and 3D configurations are lacking. It also implies that reaching an agreement to the level of 1D RT computations using state-ofthe-art computer equipment is unrealistic. A previous attempt to test 2D RT calculations has been made by Men'shchikov \& Henning (1997). They compare results from their approximate method with those of a fully-2D program (Efstathiou \& Rowan-Robinson 1990) applying the same geometry.

Here, we propose to test the behaviour of five different RT codes in a well defined 2D configuration, point out advantages and disadvantages of the various techniques applied to solve the RT problem and provide benchmark solutions for the verification of continuum RT codes. As modelling sources with high optical depth and strong scattering is the challenge of multi-dimensional RT codes, we explicitly include a test case at the limit of the current computational capabilities. In Sect. 2 we briefly introduce the RT problem and we define our test case. Sect. 3 is devoted to the description of the codes we used and to explain their differences. Solutions for the dust temperature and emerging SEDs are presented in Sect. 4. In the last section we discuss our results.

\section{Benchmark problem}

\subsection{The RT problem}

Solving the RT problem means to determine the intensity $I_{\lambda}(\boldsymbol{x}, \boldsymbol{n})$ of the radiation field at each point $\boldsymbol{x}$ and direction $\boldsymbol{n}$ of the model geometry and at each wavelength $\lambda$. This is achieved by solving the stationary transfer equation

$$
\begin{aligned}
\boldsymbol{n} \nabla_{\boldsymbol{x}} I_{\lambda}(\boldsymbol{x}, \boldsymbol{n})= & -\left[\kappa^{\mathrm{abs}}(\lambda, \boldsymbol{x})+\kappa^{\mathrm{sca}}(\lambda, \boldsymbol{x})\right] I_{\lambda}(\boldsymbol{x}, \boldsymbol{n}) \\
& +\kappa^{\mathrm{abs}}(\lambda, \boldsymbol{x}) B_{\lambda}[T(\boldsymbol{x})] \\
& +\frac{\kappa^{\mathrm{sca}}(\lambda, \boldsymbol{x})}{4 \pi} \int_{\Omega} \mathrm{d} \Omega^{\prime} p\left(\lambda, \boldsymbol{n}, \boldsymbol{n}^{\prime}\right) I_{\lambda}\left(\boldsymbol{x}, \boldsymbol{n}^{\prime}\right) \\
& +E_{\lambda}(\boldsymbol{x}, \boldsymbol{n})
\end{aligned}
$$

where $\kappa^{\text {abs }}(\lambda, \boldsymbol{x})$ and $\kappa^{\text {sca }}(\lambda, \boldsymbol{x})$ are the absorption and scattering coefficients of the particles, respectively. The quantity $p\left(\lambda, \boldsymbol{n}, \boldsymbol{n}^{\prime}\right)$ denotes the probability that radiation is scattered from the direction $\boldsymbol{n}^{\prime}$ into $\boldsymbol{n}, \Omega$ is the solid angle, $B_{\lambda}$ is the Planck function, and $T$ is the temperature. The index $\lambda$ denotes that the quantity is defined per wavelength interval. $E_{\lambda}(\boldsymbol{x}, \boldsymbol{n})$ represents all internal radiation sources such as viscous heating or cosmic rays. For the sake of simplicity, we only consider one dust component of specific size and chemical composition. In addition, we do not discuss the polarization state of the radiation field and consider the intensity only.

If spherical symmetry in the particle distribution and the sources of radiation is assumed, the integro-differential Eq. (1) becomes a function of 3 variables, already difficult to solve even for a given dust temperature $T(\boldsymbol{x})$. In the case of spatial $2 \mathrm{D}$ configurations (axial-symmetric disks, tori), we have to deal with 5 variables. Moreover, the coupling between the radiation

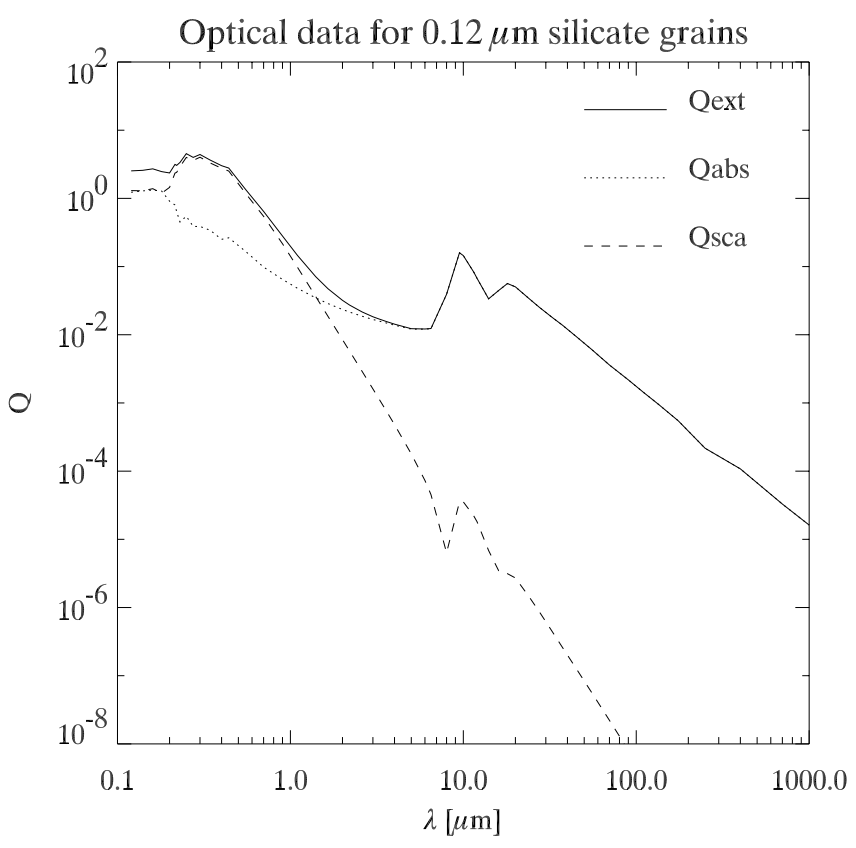

Fig. 1. Optical data for spherical astronomical silicate grains having a radius of $0.12 \mu \mathrm{m}$ (Draine \& Lee 1984). Note that scattering dominates between 0.2 and $1 \mu \mathrm{m}$ for this type of grains.

field and the dust temperature requires the simultaneous consideration of the balance equation for the local energy density at point $\boldsymbol{x}$

$$
\int_{0}^{\infty} \mathrm{d} \lambda Q_{\lambda}^{\mathrm{abs}} B_{\lambda}\left[T_{\mathrm{rad}}(\boldsymbol{x})\right]=\int_{0}^{\infty} \mathrm{d} \lambda Q_{\lambda}^{\mathrm{abs}} \frac{1}{4 \pi} \int_{\Omega} \mathrm{d} \Omega^{\prime} I_{\lambda}\left(\boldsymbol{x}, \boldsymbol{n}^{\prime}\right)
$$

to calculate intensity and temperature self-consistently. Here, $Q^{\text {abs }}(\lambda)$ is the absorption efficiency factor, while $T_{\text {rad }}$ is the temperature arising from radiative heating. Additional heating sources can contribute to the temperature with

$T(\boldsymbol{x})=T_{\text {rad }}(\boldsymbol{x})+T_{\text {heat }}(\boldsymbol{x})$.

\subsection{Model definition}

We consider the general astrophysical case of a star embedded in a circumstellar disk with an inner cavity free of dust. We assume that the star is point-like, located at the center of the configuration and radiating as a black body at the same temperature as the Sun. The disk is made of spherical astronomical silicate grains, having a radius of $0.12 \mu \mathrm{m}$ and a density of $3.6 \mathrm{~g} \mathrm{~cm}^{-3}$ (optical data are taken from Draine \& Lee $1984^{3}$, see also Fig. 1). The disk radially extends to a maximum distance of $1000 \mathrm{AU}$ from the central star. Since the correct determination of the sublimation radius is quite a difficult problem, we fix the inner radius to $1 \mathrm{AU}$. This guarantees a maximum dust temperature less than $1000 \mathrm{~K}$, even in the case of high optical depth. The density structure is that of a massless (in relation to the central star) Keplerian disk having no cutoff at a certain

\footnotetext{
3 downloadable from:

http://www .mpia.de/PSF/PSFpages/RT/benchmark . html
} 
opening angle. This implies that the radiative transfer has to be simulated both in the optically thick disk and in the optically thin envelope. The disk geometry and density structure are similar to those described by Chiang \& Goldreich $(1997,1999)$ and successfully applied to study passive disks around T Tauri stars (Natta et al. 2000). The density distribution provides a steepdensity gradient in the inner part of the disk which could give rise to numerical problems when solving the RT equation. This turns out to be an advantage for RT comparison since it allows to test the codes' behaviour under extreme conditions. The density distribution we adopt has the following form

$\rho(r, z)=\rho_{0} \times f_{1}(r) \times f_{2}(z / h(r))$

$f_{1}(r)=\left(r / r_{\mathrm{d}}\right)^{-1.0}$

$f_{2}(r)=\exp \left(-\pi / 4 \times(z / h(r))^{2}\right)$

$h(r)=z_{\mathrm{d}} \times\left(r / r_{\mathrm{d}}\right)^{1.125}$

with $r$ being the distance from the central star in the disk midplane $\left(\sqrt{x^{2}+y^{2}}\right)$ and $z$ the distance from the midplane. Here $r_{\mathrm{d}}$ is half of the disk outer radius $\left(R_{\mathrm{out}} / 2\right)$ and $z_{\mathrm{d}}$ one fourth of $r_{\mathrm{d}}\left(R_{\text {out }} / 8\right)$. Note that the disk is slightly flared, i.e., the disk opening angle $h(r) / r$ is exponentially increasing with the distance from the star. The term $f_{1}$ provides the radial dependence of the density distribution. In protoplanetary disks, the volume density is usually proportional to $r^{-\alpha}$ with $\alpha$ in the range $(1.8 \div 2.8)$ (e.g. Wood et al. 2002; Cotera et al. 2001). For this benchmark we use $\alpha=1$ in order to save CPU time. Both $f_{1}$ and $f_{2}$ remain unchanged while $\rho_{0}$ is chosen so to define different optical depths. We perform calculations for four values of visual $(\lambda=550 \mathrm{~nm})$ optical depth, namely $\tau_{\mathrm{v}}=0.1,1,10$, 100. The optical depth, as seen from the centre, is calculated along the disk midplane. Since most of the dust is confined in the midplane, the optical depths we refer to are the highest in each model. The test case $\tau_{\mathrm{v}}=100$ is at the limit of our current computational capabilities. The resulting total dust mass for the model with $\tau_{\mathrm{v}}=1(100)$ is $1.1 \times 10^{-6} M_{\odot}\left(1.1 \times 10^{-4} M_{\odot}\right)$. The density structure perpendicular to the disk midplane is shown for the same model in Fig. 2. The RT is calculated for 61 wavelengths being distributed nearly equidistantly on a logarithmic scale from $0.12-2000 \mu \mathrm{m}$. These 61 wavelengths define the frequency resolution of our computations. In Sect. 4.4 we also compare two Monte Carlo (MC) codes on a grid with two times more wavelengths and we discuss the effect of the frequency resolution on the $2 \mathrm{D}$ benchmark. Since anisotropic scattering is not included in all codes, we consider the scattering as isotropic. Symbols and values of the model parameters are summarized in Table 1 for more clarity.

\section{RT simulations}

\subsection{Methods}

Similar to hydrodynamical simulations, we can distinguish particle (Monte Carlo) and grid-based methods to solve the RT equation numerically (Henning 2001).

In MC simulations the radiation field is partitioned in equal-energy, monochromatic "photon packets" that are emitted stochastically both by the source and by the surrounding
Table 1. Model parameters.

\begin{tabular}{lll}
\hline \hline Symbol & Meaning & Value \\
\hline$M_{*}$ & Stellar mass & $1 M_{\odot}$ \\
$R_{*}$ & Stellar radius & $1 R_{\odot}$ \\
$T_{*}$ & Stellar effective temperature & $5800 \mathrm{~K}$ \\
$R_{\text {out }}$ & Outer disk radius & $1000 \mathrm{AU}$ \\
$R_{\text {in }}$ & Inner disk radius & $1 \mathrm{AU}$ \\
$z_{\mathrm{d}}$ & Disk height & $125 \mathrm{AU}$ \\
$a$ & Grain radius & $0.12 \mu \mathrm{m}$ \\
$\rho_{\mathrm{g}}$ & Grain density & $3.6 \mathrm{~g} \mathrm{~cm}^{-3}$ \\
$\tau_{\mathrm{v}}$ & Optical depth at $550 \mathrm{~nm}$ & $0.1,1,10,100$ \\
\hline
\end{tabular}

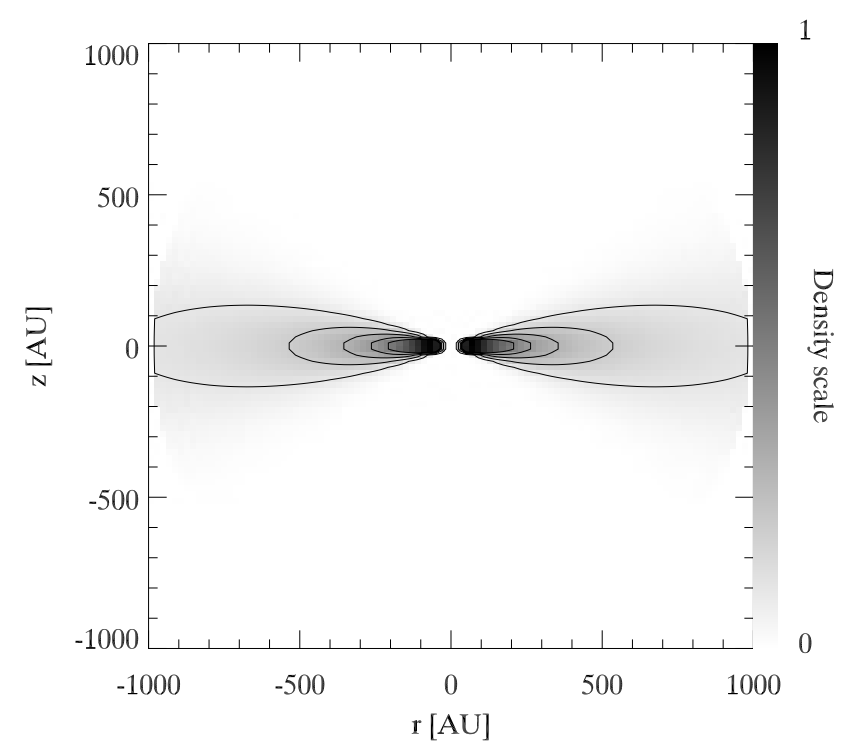

Fig. 2. Density structure perpendicular to the disk midplane and centered on the star for the model with $\tau_{\mathrm{v}}=1$. Values are normalized to the maximum density. The contours provide $0.10,0.19,0.28,0.38$, $0.48 \%$ of the maximum.

envelope. The optical depth determines the location at which the packets interact while their albedo defines the probability of either scattering or absorption. In the original scheme (scheme 1) the source and the envelope photon packets are emitted separately. At first the grains re-emit according to the absorbed source radiation. Then dust reemission takes also into account the envelope emission radiation field. Reemission by the dust is repeated as long as the difference between the input and the output energy is larger than a chosen threshold. However, the dust reemission, i.e. the repetition of the Monte Carlo experiment, is time consuming. An alternative possibility (scheme 2) is to store all radiation exchanges within the envelope. In this case the Monte Carlo experiment can be carried out once for all $^{4}$, but a large amount of computer memory is needed. A drawback of these two schemes is that the input luminosity is not automatically conserved during the simulation. This becomes a serious problem for configurations with very high optical depths which therefore usually need

\footnotetext{
${ }^{4}$ This is only valid for opacities explicitely independent on the temperature.
} 
Table 2. Main features of the codes.

\begin{tabular}{|c|c|c|c|c|c|}
\hline Feature & MC3D & MCTRANSF & RADICAL & RADMC & STEINRAY \\
\hline $3 \mathrm{D}$ & + & & & & + \\
\hline Anisotropic scattering & + & + & & + & + \\
\hline Arbitrary grid geometry & + & + & & & \\
\hline Grain size distribution & + & + & + & + & + \\
\hline Multiple dust species & + & & + & + & + \\
\hline Images & + & + & + & + & + \\
\hline Polarization maps & + & & & & \\
\hline Global error control & & & + & & + \\
\hline Multiple/extended heating sources & + & & + & & + \\
\hline Dust evaporation included & & + & + & & \\
\hline Acceleration for high $\tau$ (ALI/Ng/other) & + & + & + & & + \\
\hline Parallel version & & + & + & & \\
\hline
\end{tabular}

a larger number of iterations. A solution has been found by Bjorkman \& Wood (2001) in the so-called immediate reemission (scheme 3). In this case only source photon packets are emitted and followed in their interaction locations. When a packet is absorbed, its energy is added to the envelope and a new packet is emitted immediately at a frequency which takes into account the modified envelope temperature. This method does not require any iteration and implicitly conserves the total energy. Another improvement of the standard MC procedure has been proposed by Lucy (1999) to treat extremely optically thin configurations. This approach considers the absorption not only at the end points of the photon path but also in between.

Grid-based codes solve the RT equation on a discrete spatial grid. The grid can be either determined during the simulation or generated before starting the computation. The 2 RT grid-based codes we compare, namely RADICAL and STEINRAY, use the second approach (see Sects. 3.2.3 and 3.2.5). Among the schemes applied to solve the RT problem two are the most used: the so-called "Lambda Iteration" (see e.g. Collison \& Fix 1991; Efstathiou \& Rowan-Robinson 1991 ) and the "Variable Eddington Tensor" (Mihalas \& Mihalas 1984; Malbet \& Bertout 1991; Stone et al. 1992; Kikuchi et al. 2002; Dullemond et al. 2002; Dullemond 2003). The "Lambda Iteration" mode suffers from the same convergence problems as the standard MC which is based on scheme 1. Thus, the improved "Accelerated Lambda Iteration" (e.g. Rybicki \& Hummer 1991) method is more widely applied. The "Variable Eddington Tensor" mode is more robust than the "Lambda Iteration" and usually converges faster. Moreover, it has been proven that it works properly even at extremely high optical depths. Integration of the formal RT equation can be done in a straightforward way by applying the "Long Characteristics" algorithm. This method is accurate but turns out to be costly in CPU time. A more efficient way is based on the "Short Characteristics" algorithm (Mihalas et al. 1978; Kunasz \& Auer 1988).

Each of the solution algorithms has its advantages and drawbacks. In MC methods, a photon is propagated through the calculation domain and its scattering, absorption, and re-emission are tracked in detail. This allows to treat very complicated spatial distributions, arbitrary scattering functions and polarization. Drawback is the presence of a random noise in the results. This noise can be reduced by increasing the number of used photon packages and by including deterministic elements in the MC experiment (Niccolini et al. 2003). Gridbased solvers are less flexible than MC codes but have the advantage not to involve random noise.

\subsection{Code description}

In the following sections we briefly describe the RT codes participating in the 2D benchmark. A summary of their main features is provided in Table 2.

\subsubsection{MC3D}

MC3D is a 3D continuum RT code. It is based on the MC method and solves the RT problem self-consistently. MC3D is designed for the simulation of dust temperatures in arbitrary dust/electron configurations and the resulting observables: spectral energy distributions, wavelength-dependent images and polarization maps.

For the estimation of temperatures either the standard scheme 1 (see Wolf et al. 1999b; Wolf \& Henning 2000) or the immediate reemission concept (scheme 3) can be applied. For this benchmark project, the scheme 3 is used to treat properly the more optically thick models. Optically very thin configurations, such as the atmosphere/envelope described in Sect. 2.2, are easily computed by the method proposed by Lucy (1999). Furthermore, the efficiency of MC3D is increased by (a) the fast photon transfer and (b) wavelength range selection concept (see Wolf \& Henning 2000 for details), and (c) the enforced scattering mechanism as described by Cashwell \& Everett (1959).

Previous applications of MC3D cover feasibility studies of extrasolar planet detections (Wolf et al. 2002), the RT in the clumpy circumstellar environment of YSOs (Wolf et al. 1998), polarization studies of T Tauri stars (Wolf et al. 2001a), AGN polarization models (Wolf \& Henning 1999a), a solution for the multiple scattering of polarized radiation by non-spherical 
grains (Wolf et al. 2002), and the inverse RT based on the MC method (Wolf 2001b). Executables of MC3D (V2) can be downloaded for several model geometries and platforms from: http://www . mpia-hd.mpg. de/FRINGE/SOFTWARE/mc3d/.

\subsubsection{MCTRANSF}

MCTRANSF solves multi-dimensional continuum RT problems in dusty media by means of a MC method. It has been originally developed by Lopez et al. (1995). So far, the code has been used for the empirical modelling of several circumstellar envelopes of post AGB-stars of different types (e.g. Lopez \& Perrin 2000), including multi-scattering effects.

Currently, only spherical symmetric (1D) and axisymmetric (2D) problems can be considered, but an extension to 3D is possible and straightforward. Several improvements of the standard MC procedure have been recently included (Niccolini et al. 2003) in order to avoid the usual increase of the noise level which typically occurs in extremely optically thin or optically thick situations. The concept suggested by Lucy (1999) is implemented to treat very optically thin cases. Optically thick configurations are tackled by the inclusion of several deterministic elements for the treatment of the absorption during the photon propagation phase, forcing the absorption to take place all along the rays. The temperature structure of the medium in radiative equilibrium is found by applying scheme 2 . The convergence is found to be rapid, even in optically thick situations, but needs a large amount of computer memory, because the primary MC information must be stored source-dependently.

As a result of the combination of all these measures, MCTRANSF is capable to simultaneously model optically thin and optically thick parts of the model volume with about the same accuracy. Thus, both clumpy media and discontinuous opacity structures can be handled. MCTRANSF is able to arrive at numerical solutions for RT problems even in case of very large optical depths (e.g. for disk configurations). Parallelised versions of the code have been developed for a Cray T3E 1200 and for systems supporting the OpenMP application program interface. All these versions use shared memory systems.

\subsubsection{RADICAL}

The core of the code RADICAL is a lambda operator subroutine based on the method of "Short Characteristics", implemented on a polar grid by Dullemond \& Turolla (2000). Using this subroutine as the main driver, RADICAL offers two modes of operation: a simple Lambda Iteration mode and a Variable Eddington Tensor mode. In this paper we use the latter because of its faster convergence and capability of treating high optical depths. The Variable Eddington Tensor method is implemented in RADICAL as follows (Dullemond 2003). First, the primary stellar radiation field is propagated from the star outwards into the disk. Dust scattering is included in a MC fashion. The energy absorbed by the disk in each grid-cell is then re-emitted as infrared (IR) radiation, which is treated as a separate radiation field. The $2 \mathrm{D}$ transfer solution for this secondary radiation field is found by solving the frequency-integrated moment equations. The closure for these equations is based on the variable Eddington tensors and mean opacities computed with the Short Characteristics method of Dullemond \& Turolla. At the end of the calculation a global check on flux conservation is made. For all the models discussed in this paper the error remained within $2 \%$.

\subsubsection{RADMC}

RADMC is an MC code based on scheme 3. However, the original method of Bjorkman \& Wood produces very noisy temperature profiles in regions of low optical depth, and requires a large number of photons $\left(N \sim 10^{7}\right)$ for a smooth SED. These disadvantages have been solved in RADMC by treating absorption partly as a continuous process (Lucy 1999), and using the resulting smooth temperature profiles with a ray-tracing code to produce images and SEDs. These images and SEDs have a low noise level even for relatively few photon packages $\left(N \sim 10^{5}\right)$. In addition, the frequency grid used for RADMC is not bound by the constraints set in the original method. This improved Bjorkman \& Wood method works well at all optical depths, but may become slow in cases where the optical depth is very large ( $\tau_{\mathrm{v}}$ about 1000$)$. For the test cases in this paper the optical depths are low enough that this problem does not play a role. For more information on the code, see http://wWw.mpa-garching.mpg.de/PUBLICATIONS/ DATA/radtrans/radmc/

\subsubsection{STEINRAY}

STEINRAY is a grid-based code which solves the full 3D continuum RT problem. A combination of ray-tracing and finite differencing of 2 nd order on adaptive multi-frequency photon transport grids is applied. Steinacker et al. (2002a) have shown that the use of 3rd order finite differencing is too timeconsuming for 3D RT, while 1st order schemes introduce an unacceptable degree of numerical diffusion to the solution.

The spatial grids are generated using an algorithm described in Steinacker et al. (2002c). They are adaptive and optimized to minimize the 1 st order discretization error hence guaranteeing global error control for solutions of radiative transfer problems on the grid. Since the use of one single grid for all frequencies leads to large discretization errors, STEINRAY calculates individual grids for each frequency to use the global error control of the grid generation method. Minimization of the grid point number is possible in regions where the optical depth becomes large allowing for treatment of applications with optical depth of any value. Contrary to former treatments, the full frequency-dependent problem is solved without any flux approximation and for arbitrary scattering properties of the dust. For the direction discretization, equally spaced nodes on the unit sphere are used along with corresponding weights for the integration derived by evaluating special Gegenbauer polynomials in Steinacker et al. (1996). The temperature distribution is calculated by an Accelerated Lambda Iteration between the 
Table 3. Resolution and number of photons for the different test cases.

\begin{tabular}{lllllll}
\hline \hline $\begin{array}{l}\text { Code } \\
\text { name }\end{array}$ & $\# r$ & $\begin{array}{l}\Delta r \\
{[\mathrm{AU}]}\end{array}$ & $\# \theta$ & $\begin{array}{l}\Delta \theta \\
{\left[{ }^{\circ}\right]}\end{array}$ & $\begin{array}{l}\text { \# Phot } \\
{\left[\times 10^{6}\right]}\end{array}$ & $\begin{array}{l}\text { Test case } \\
\tau_{550 \mathrm{~nm}}\end{array}$ \\
\hline MC3D & 55 & $0.03-141$ & 121 & 1.5 & 244 & $0.1,1,10$ \\
MC3D & $10^{3}$ & $0.07-4.1$ & 121 & 1.5 & 244 & 100 \\
\hline MCTRANSF & 48 & $0.17-125$ & 40 & 4.5 & 1000 & 0.1 \\
MCTRANSF & 48 & $0.17-125$ & 40 & 4.5 & 800 & 1 \\
MCTRANSF & 46 & $0.18-130$ & 46 & $2.8-5.3$ & 1000 & 10 \\
MCTRANSF & 46 & $0.18-130$ & 46 & $2.8-5.3$ & 500 & 100 \\
\hline RADICAL & 60 & $0.03-116$ & 62 & $1.6-8.3$ & & $0.1-100$ \\
\hline RADMC & 60 & $0.03-116$ & 62 & $1.6-8.3$ & 10 & $0.1-100$ \\
\hline STEINRAY & 61 & $0.12-109$ & 61 & 1.3 & & $0.1-100$ \\
\hline
\end{tabular}

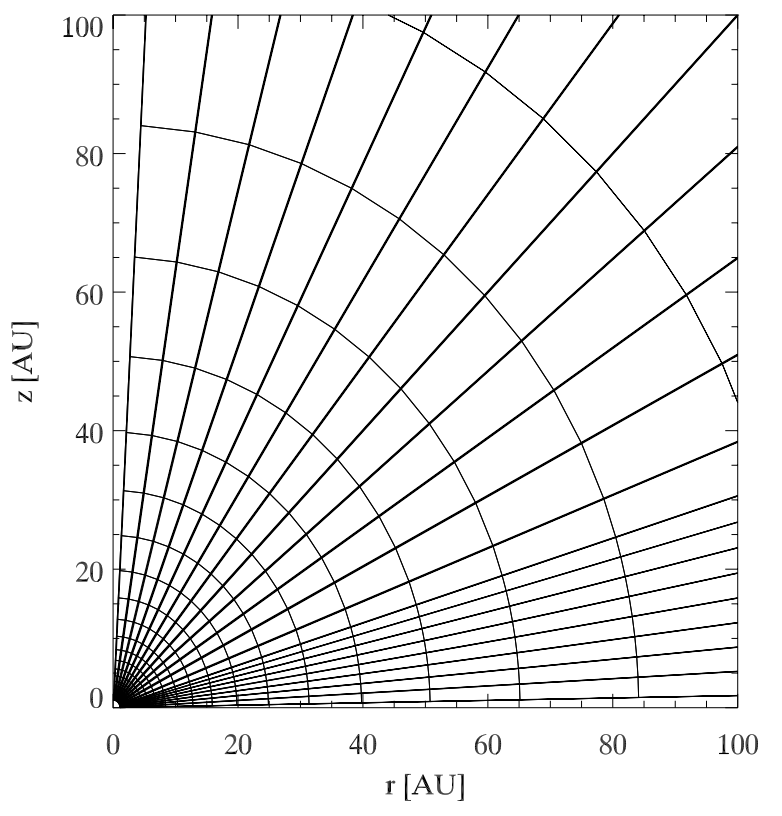

Fig. 3. Grid adopted by MCTRANSF to store the temperature resulting from the RT simulations. Similar spherical grids are also used by the other codes.

radiative transfer equation and the local balance equation. The program is designed to provide spatially resolved images and spectra of complex 3D dust distributions and allows for multiple internal and external sources (Steinacker et al. 2003).

Recently, a 2D version of the program has been developed and was used for this benchmark. The grids are similar to the spherical grids shown in Fig. 3. The 2D version uses ray-tracing to solve for the intensity in all directions.

\subsection{Reliability of the codes in $1 D$}

All the RT codes participating in the benchmark have been already tested in 1D spherically symmetric configurations. Results from MC3D have been compared with those calculated with the RT code of Chini et al. (1986) and with the code of Menshchikov \& Henning (1997). In both cases differences below $1 \%$ even in the case of high optical depth have been found (Wolf et al. 1999b). MCTRANSF has been tested (Niccolini et al. 2003) against the 1D code written by Thibaut Le Bertre and based on the work of Leung (1976). For the case of optical depth equal to 10 at $1 \mu \mathrm{m}$ (with $\kappa \propto \frac{1}{\lambda}$, and isotropic scattering) the agreement between MCTRANSF and le Bertre's code is better than $1 \%$. In the case of RADICAL, tests have been performed by comparing the results with those from TRANSPHERE $^{5}$, a 1-D variable eddington factor code tested against the similar "code 1" of Ivezic et al. (1997). Steinacker et al. (2003) used the 1D benchmark provided by Ivezic et al. (1997) to test the 3D version of STEINRAY. Agreement below $1 \%$ has been found both for the emerging temperature and SEDs.

\subsection{Details on the $2 D R T$ computation}

The three MC codes involved in the 2D benchmark, namely MC3D (Sect. 3.2.1) and MCTRANSF (Sect. 3.2.2) and RADMC (Sect. 3.2.4), choose a spherical grid to store the temperature resulting from the RT simulations. Radially the steps are logarithmic in order to properly resolve the innermost dense region of the disk. The number of radial points is kept below 100 for all the codes but MC3D, for which we tried a two weeks long computation for the most optically thick case (see Table 3). Differences in the SED between this computation and the one with 55 radial points and lower number of photons are discussed in Sect. 4.4. MC3D adopts an equally spaced grid in vertical direction $\left(1.5^{\circ}\right.$ resolution, see Table 3$)$, while MCTRANSF and RADMC choose a resolution decreasing with the distance from the disk midplane (see Fig. 3). Similar grid geometries are also used by the two grid-based codes RADICAL and STEINRAY. RADICAL (Sect. 3.2.3) makes use of the same grid as RADMC while STEINRAY (Sect. 3.2.5) has comparable resolution in vertical direction but larger cells in the inner disk (0.12 AU, see Table 3$)$.

We note that the resolution given in Table 3 , together with the number of photons for the MC codes, are at the limit of the computing capabilities for most of the codes. MC3D needs about 1 Gby memory. The temperature resulting from these test cases is obtained in 1-2 days. Computing the SED requires about a week for all the models, but for the most optically thick one for which we try a longer computation. MCTRANSF needs

\footnotetext{
5 http://www.mpa-garching.mpg.de/PUBLICATIONS/ DATA/radtrans/
} 
a large amount of computer memory to store all radiation exchanges. The necessary memory goes as the square of the number of cells: $46 \times 46$ cells is actually the technical limit ( 4 Gby) on our 4-processors (ev67 at $1 \mathrm{GHz}$ ) HP-compaq ES45 server. The runtime for the most optically thick case is about 2 weeks. Results from RADICAL and RADMC are obtained in less than a day for all the test cases. However, the actual spatial resolution of RADICAL cannot be doubled due to not sufficient computer memory. To produce the final spectrum RADMC uses the ray-tracing module from RADICAL, and for that reason is also limited to the maximum spatial resolution that can be achieved by RADICAL. This is a mere technical problem. In the $2 \mathrm{D}$ version of STEINRAY, the code uses about 1 Gby memory. To obtain convergence in the temperature iteration of $1 \%$ for the case $\tau_{\mathrm{v}}=100$, the code runtime is about a week.

\section{Results}

\subsection{Approximate solution for optically thin configurations}

In the case of configurations being optically thin for all relevant wavelengths, heating of the dust particles is dominated by the stellar radiation. When re-emission of the dust particles can be neglected and scattering is only included as extinction term, the dust temperature can be easily determined from Eq. (2) without any coupling to Eq. (1). We use this approximate semianalytical solution to test independently our RT computations for the most optically thin case and to check the correctness of the density setup in the other more optically thick models (see Sects. 4.2 and 4.3).

The assumptions we discussed above simplify Eq. (2) in the following way

$$
\begin{aligned}
\int_{\lambda_{\min }}^{\lambda_{\max }} \mathrm{d} \lambda B_{\lambda}\left[T_{\mathrm{d}}(R, \theta)\right] Q_{\lambda}^{\mathrm{abs}}= & \left(\frac{R_{*}}{2 R}\right)^{2} \\
& \times \int_{\lambda_{\min }}^{\lambda_{\max }} \mathrm{d} \lambda B_{\lambda}^{\mathrm{e}}\left(T_{*}, R, \theta\right) Q_{\lambda}^{\mathrm{abs}}
\end{aligned}
$$

with $T_{\mathrm{d}}(R, \theta)$ being the disk temperature at the location $(R, \theta)^{6}$ and $Q^{\text {abs }}(\lambda)$ the absorption efficiency factor. We perform the integration at the same wavelengths as those adopted by the RT codes (see Sect. 2.2). The term $B_{\lambda}^{\mathrm{e}}\left(T_{*}, R, \theta\right)$ represents the black body emission from the star corrected for the extinction

$B_{\lambda}^{\mathrm{e}}\left(T_{*}, R, \theta\right)=B_{\lambda}\left(T_{*}\right) \mathrm{e}^{-\pi a^{2}\left(Q_{\lambda}^{\text {abs }}+Q_{\lambda}^{\text {sca }}\right) \int_{0}^{\mathrm{R}} \mathrm{d} R^{\prime} \rho\left(R^{\prime}, \theta\right)}$

where $a$ is the dust radius, $Q^{\text {sca }}(\lambda)$ is the scattering efficiency factor and $\rho\left(R^{\prime}, \theta\right)$ is the density distribution given in Eq. (4) but here expressed in spherical coordinates. The argument of the exponent represents the optical depth $\tau_{\lambda}(R, \theta)$ at the distance $R$, $\theta$ from the central star. Once the optical depth is determined, the extincted black body emission can be substituted in Eq. (5) and the dust temperature can be easily computed. To obtain the flux density $F_{\lambda}$ at a distance equal to the stellar radius we need

${ }^{6} R$ is the distance from the central star in spherical coordinates and $\theta$ is the polar angle as measured from the disk midplane.



Fig. 4. Differences of the most optically thin model from the semianalytical solution (see Sect. 4.1). Upper panel: differences in radial temperature for an angle $\theta$ near to the disk midplane. Lower panel: differences in the SED for an almost face-on disk (disk inclination equal to $12.5^{\circ}$ ). For both panels, solid lines give the difference of MC3D, dot-dashed lines of MCTRANSF, dashed-dot-dot-dot of RADICAL, dotted lines of RADMC, and dashed lines of STEINRAY from the semi-analytical solution.

to integrate the power emitted by each grain over the entire volume. If we express the power emitted by one grain as

$P_{\lambda}^{\mathrm{g}}(R, \theta)=4 \pi a^{2} Q_{\lambda}^{\mathrm{abs}} B_{\lambda}\left[T_{\mathrm{d}}(R, \theta)\right]$

the flux density can be obtained by solving the following integrals

$F_{\lambda}=\frac{2 \pi}{4 \pi R_{*}^{2}} \int_{\mathrm{R}_{\text {in }}}^{\mathrm{R}_{\text {out }}} \int_{\theta=0}^{\pi} \mathrm{d} \theta^{\prime} \mathrm{d} R^{\prime} P_{\lambda}^{\mathrm{g}}\left(R^{\prime}, \theta^{\prime}\right) \rho\left(R^{\prime}, \theta^{\prime}\right) R^{\prime 2} \cos \left(\theta^{\prime}\right)(8)$

here the factor $2 \pi$ comes from the integration in $\phi$ which is the azimuthal angle in the $x-y$ plane.

A first rough estimate of the correctness of this approach can be done by evaluating how much the disk temperature $T_{\mathrm{d}}$ would increase because of secondary emission re-absorption events. The grain emissivity is proportional to $T_{\mathrm{d}}^{4}$ and, in case of optically thin emission, to the optical depth. Our most optically thin model has a maximum IR optical depth of 0.01 at $10 \mu \mathrm{m}$ in the disk midplane, where most of the dust is confined. If an emitted IR photon were re-absorbed by the disk, its temperature would increase by the quantity $\left(1+\tau_{\mathrm{IR}}\right)^{0.25}$. Thus, the temperature obtained by the semi-analytical approach can be considered correct within $0.26 \%$ in a first approximation. The corresponding emergent flux has an uncertainty which is about four times larger (about 1\%). However looking at the SED differences (see Fig. 4), it is clear that deviations due to scattering (treated correctly in the numerical codes) are important too. A more realistic estimate of the error on the semi-analytical approach should indeed consider the effect of scattering apart from damping the stellar flux. 


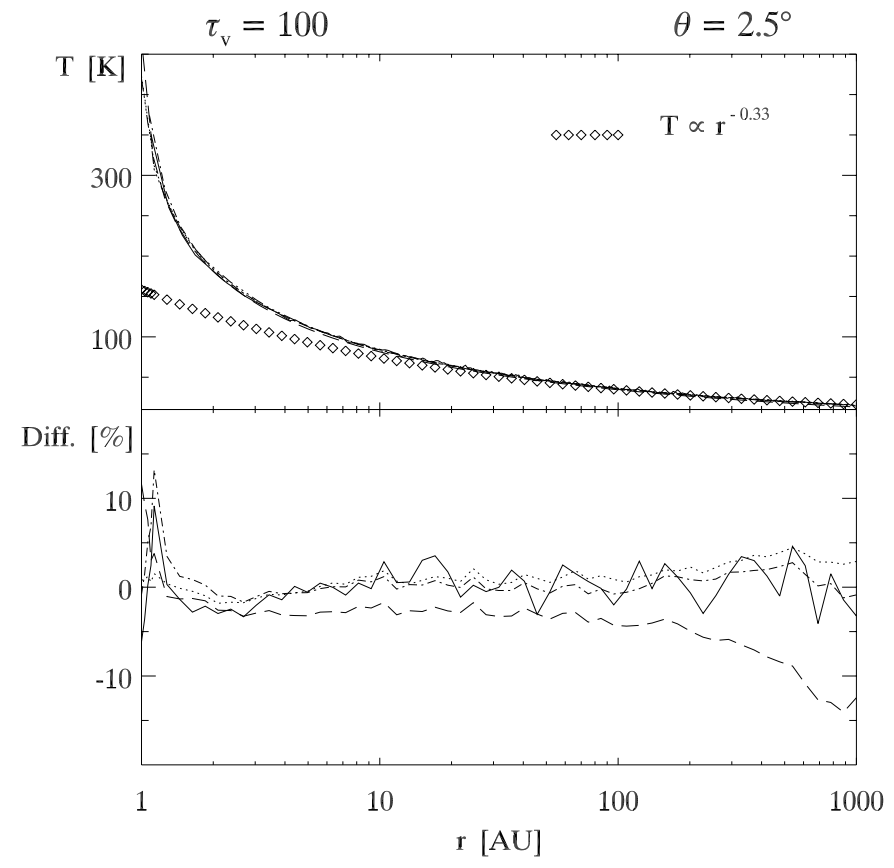

Fig. 5. Radial temperature (upper panel) and percentage of difference (lower panel) among the codes for the most optically thick model. RADICAL is taken as reference code. The radial cut is made for an angle $\theta$ near to the disk midplane. Diamonds give the radial dependence in case of long wavelengths and optically thin emission. In the upper panel, solid lines are the results from MC3D, dot-dashed lines from MCTRANSF, dashed-dot-dot-dot from RADICAL, dotted lines from RADMC and dashed lines from STEINRAY. In the lower panel, solid lines give the difference of MC3D, dot-dashed lines of MCTRANSF, dotted lines of RADMC and dashed lines of STEINRAY from RADICAL.

\subsection{Temperature}

All the codes correctly reproduce the shape of the temperature distribution, both its radial and vertical dependence.

In oder to test independently the most optically thin case (highest optical depth in the disk midplane of $\tau_{\mathrm{v}}=0.1$ ), we use the semi-analytical solution derived in Sect. 4.1. The upper panel of Fig. 4 shows the percentage of difference ${ }^{7}$ between the semi-analytical solution and any other code. Radial cuts are plotted for an angle $\theta$ near to the disk midplane where the influence of scattering is the largest. The differences between the semi-analytical solution and the RT codes is always smaller than $1 \%$.

Temperature distributions for the models with $\tau_{\mathrm{v}}=1$ and 10 agree better than $10 \%$ for all the cases we examine. The disk model with the highest optical depth is the most difficult to treat for the RT codes. In Figs. 5 and 6, we show radial and vertical cuts at the disk locations where deviations from the codes are expected to be higher, i.e. near to the disk midplane and close to the central star. The radial temperature is plotted for an angle $\theta$ equal to $2.5^{\circ}$ from the disk midplane while the vertical temperature is given for a distance equal to $2 \mathrm{AU}$ from the central star. In the upper panel of Fig. 5, we also superimpose

\footnotetext{
7 With difference we mean: $\frac{(a-b)}{b}$. Here $b$ stands for the reference solution/code while $a$ for any other code.
}



Fig. 6. Vertical temperature and percentage of difference among the codes for the most optically thick model and for a distance $r$ in the midplane equal to $2 \mathrm{AU}$ from the central star. RADICAL is taken as reference code. The nomenclature is the same as for Fig. 5 except for the diamonds which give the temperature behaviour following the semi-analytical approach described in Sect. 4.1. Note that all the RT codes have the same turnover in the temperature distribution at the location predicted by the semi-analytical solution.

the temperature dependence for the optically thin regime at long wavelengths. In this regime the temperature distribution depends only on the dust properties and can be approximated by $T(r) \propto r^{-2 /(4+\beta)}$ (Evans 1994). Here $\beta$ corresponds to the index of the dust absorption coefficient at low frequencies $\left(\kappa_{v}^{\text {abs }} \propto \nu^{\beta}\right)$. For Draine \& Lee silicates $\beta$ is equal to 2 , leading to an exponent of -0.33 in the temperature relation.

The upper panel of Fig. 6 provides (in diamonds) the vertical temperature profile from the semi-analytical approach described in Sect. 4.1. The semi-analytical solution has the turnover point from optically thick to optically thin (the place where the temperature suddenly starts to drop) around $19^{\circ}$ from the midplane. Since the solution provided by the RT codes should have the same behaviour, we used the semi-analytical approximation to check the correctness of the density setup. The disk midplane calculated with the semi-analytical approach is naturally cooler than the real disk because the approximation neglects heating of the disk from dust re-emission. The outer regions of the real disks are also warmer because of those photons scattered far from the midplane. At a distance $r$ of $2 \mathrm{AU}$ from the star and exactly in the midplane the real temperature is a factor of about 1.3 higher than that given by the semi-analytical solution.

The lower panels of Figs. 5 and 6 provide the percentage of difference among the codes taking RADICAL as reference code. The radial temperatures agree better than $5 \%$ in most of the disk, going from 1.2 AU to $200 \mathrm{AU}$. Around 1.1 AU 

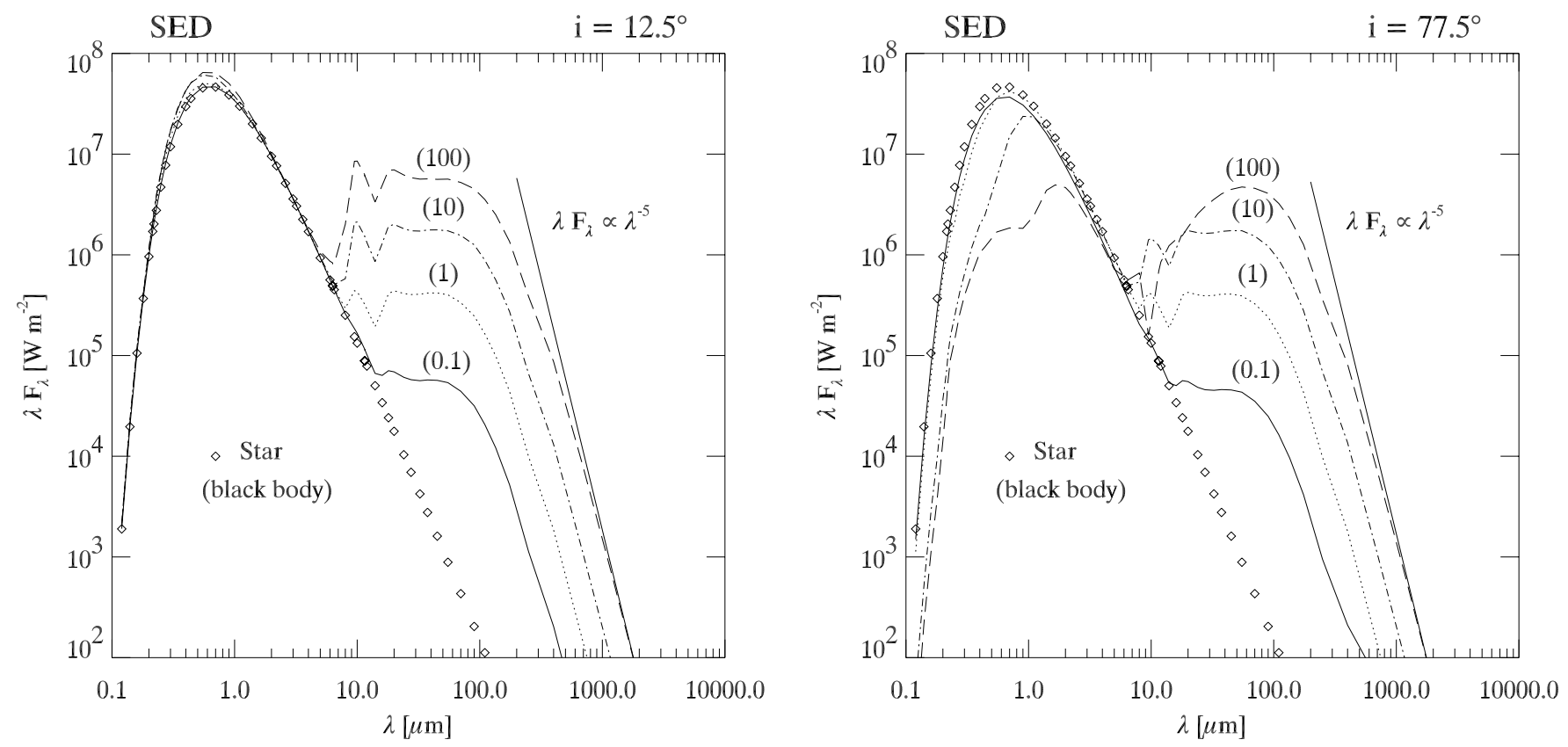

Fig. 7. SED for two disk inclinations $i$ as given on top of each panel. Each curve provides the mean value of the five RT simulations for the four computed models with different optical depth. The midplane optical depth is given in parenthesis labeling each curve. In both panels solid lines show results for the most optically thin disk, dotted lines for a disk having $\tau_{\mathrm{v}}=1$, dot-dashed lines for a disk with $\tau_{\mathrm{v}}=10$ and dashed lines for the most optically thick model. Diamonds provide the black-body emission from the naked star. The slope of the SED at long wavelengths depends only on the dust properties and is plotted in each panel with a solid line.

MCTRANSF deviates slightly more than $10 \%$. STEINRAY shows $10 \%$ deviations at the inner boundary and slightly higher deviations (but always less than 15\%) far from the star, at about $900 \mathrm{AU}$. The vertical cut at 2 AU shows an agreement better than $2.5 \%$ till $10^{\circ}$ from the disk midplane. Closer to the disk midplane, deviations are larger for MC3D and STEINRAY but always smaller than $4 \%$.

\subsection{Spectral energy distribution}

The emerging spectra for the four models having different optical depths are shown in Fig. 7 at two disk inclinations. The left panel provides the results for an almost face-on disk (disk inclination $i$ equal to $12.5^{\circ}$ ) while the right panel gives the result for an almost edge-on disk $\left(i=77.5^{\circ}\right)$. Each curve represents the mean value of the five RT simulations for the specific model, whose optical depth is given in parenthesis above the curve. On the $y$ axis, we plot $\lambda F_{\lambda}$ in $\left[\mathrm{W} \mathrm{m}^{-2}\right]$ where $F_{\lambda}$ is the flux density at a distance equal to the star radius. We also superimpose in diamonds the black body radiation arising from the star in order to visualize how efficiently the circumstellar disk reprocesses the stellar energy. We note that all the codes have the correct slope at long wavelengths. This slope depends only on the dust properties and is plotted as solid line in both panels $\left(\lambda F_{\lambda} \propto \lambda^{-5}\right)$. At $0.55 \mu \mathrm{m}$ the drop in luminosity amounts to about a factor of 20 going from the most optically thin to the most optically thick model and for a disk inclination of $77.5^{\circ}$.

Since the differences among the codes are too small to be visible in a logarithmic plot, we provide separately the percentage of difference for the four models and for three disk inclinations (see Fig. 8). RADICAL has been chosen as reference code. For the most optically thin case, we also compare our results with the semi-analytical approach (see Fig. 4). We find that the agreement of the codes with the semi-analytical solution is always better than $8 \%$, with the largest deviations around 0.3 and $40 \mu \mathrm{m}$. In the range $0.2-0.7 \mu \mathrm{m}$ all the codes predict higher flux in comparison to the semi-analytical solution, while between 10-200 $\mu \mathrm{m}$ a lower flux is obtained. These deviations arise because the semi-analytical approach includes scattering only as an extinction term. From the numerical RT calculations it is clear that some photons are scattered thus enhancing the flux between 0.2 and $0.7 \mu \mathrm{m}$. We note that this wavelength range is exactly where small astronomical silicate grains have the largest scattering efficiency (see Fig. 1). Therefore, deviations peaking at $0.3 \mu \mathrm{m}$ are simply explained by the particular optical data chosen for this benchmark. Those photons which are scattered cannot contribute to heat the disk. This explains why RT codes predict a lack of emission at longer wavelengths. To understand why the largest deficit of photons is around $40-50 \mu \mathrm{m}$, we first compute the temperature at which most of the disk mass emits (mass average temperature) and then the corresponding wavelength. For the wavelength calculation we need to take into account the grain emissivity (Evans 1994). We find a mass average temperature of $40 \mathrm{~K}$ which translates into a wavelength of $50 \mu \mathrm{m}$ at the maximum emission. This wavelength is well in agreement with the deviations shown in Fig. 4. For comparison, the RT codes agree better than $1.5 \%$ at wavelengths shorter than $10 \mu \mathrm{m}$ for this particular test case $\left(\theta=12.5^{\circ}\right)$. At longer wavelengths the results show a bit more scatter but the agreement is always better than $3 \%$. 



Fig. 8. Percentage of difference in the SED between the codes. RADICAL is taken as reference code. Solid lines give the difference between MC3D and RADICAL, dot-dashed lines between MCTRANSF and RADICAL, dotted lines between RADMC and RADICAL and dashed lines between STEINRAY and RADICAL.

In Fig. 8 first panel, we also show the percentage of difference for two other disk inclinations, namely $42.5^{\circ}$ and $77.5^{\circ}$. In both cases the agreement is better than $2 \%$ at all wavelengths for all the codes but MCTRANSF, for which slightly higher deviations (about 2.5\%) are present at longer wavelengths.

As the optical depth in the midplane increases, the RT problem becomes more difficult to solve. Because of the chosen disk geometry, most of the disk mass is located near to the midplane and close to the disk inner boundary. Our comparison shows that agreement among the codes is always better for an almost face-on case and $42.5^{\circ}$ disk inclination (first two panels of Fig. 8), than for an almost edge-on disk (lower panels of Fig. 8). For the models with $\tau_{\mathrm{v}}=1$ deviations among the codes are smaller than $9 \%$. For the disk with midplane optical depth of 10 and 100 and inclinations of 12.5 and $42.5^{\circ}$, differences do not exceed $10 \%$. For the almost edge-on configurations the most difficult regions to treat are those where scattering dominates and at wavelengths around $10 \mu \mathrm{m}$. In the IR, opacities change strongly and the modified Planck emission peaks in the inner disk regions (between 1 and $2 \mathrm{AU}$ ). Therefore, the numerical simulations are particularly sensible to the resolution of the inner parts. Deviations in the IR are 
partly due to the different resolution adopted by the codes (see also Sect. 4.4). Scatter at visible and near-infrared wavelengths for MC3D, MCTRANSF, and RADMC is simply statistical noise, typical of MC simulations. This scatter becomes more prominent at high optical depths. We also note that MC3D and RADMC have the same trend for wavelengths larger than $10 \mu \mathrm{m}$ and the model with $\tau_{\mathrm{v}}=100$ : they both estimate a larger IR emission than RADICAL with peaks at $\sim 70 \mu \mathrm{m}$ for MC3D and at $\sim 100 \mu \mathrm{m}$ for RADMC. A strong deviation from the other codes is shown by STEINRAY just after the $9.8 \mu \mathrm{m}$ silicate feature. However, one should note that apart from the discussed features the overall agreement of the SEDs is better than $10 \%$ for all the codes even for the almost edge-on disk and the most optically thick test case.

\subsection{Tests for various spatial and frequency resolutions}

We used the MC code MC3D to test the dependence of our results on the grid adopted to store the emerging temperature. Since deviations due to different temperature sampling are expected to be larger for more optically thick configurations, we investigate our most optically thick test case $\tau_{\mathrm{v}}=100$. Different grids, covering radial resolutions from 2.7 AU up to $0.03 \mathrm{AU}$ in the inner disk and vertical resolutions from $1.5^{\circ}$ to $5.8^{\circ}$, have been inspected. In Fig. 9, we report results for five relevant cases. The number of radial and vertical subdivisions $(\# r$ and $\# \theta$ ), as well as the resolution $(\Delta r$ and $\Delta \theta$ ) and total number of photons (\#Phot) for these cases are provided in Table 4. The RT equation is solved for 61 wavelengths, the same assumed in all the previous simulations. The number of photons is set to $4 \times 10^{5}$ per wavelengths to limit the runtime to 2 days on a PC with 4 Gby memory, $2.4 \mathrm{GHz}$ clock. Only in one case $(\bmod 4)$ we let the computation run for about two weeks in order to reduce as much as possible the photon noise at short wavelengths. The comparison shows that the vertical spacing does not influence too much the results: we report deviations smaller than $1.5 \%$ for the three disk inclinations between the models with vertical resolution $1.5^{\circ}$ and $5.8^{\circ}$ (solid line in Fig. 9). On the other side, changes in the radial grid strongly affects the IR emission: when going from $\bmod 0$ to $\bmod 2$ results still do not differ more than $5 \%$ but the coarse inner grid of $\bmod 3$ causes deviations larger than $20 \%$. Model mod 4 is the state-of-the-art for our computer capabilities. The grid has a good resolution also in the outer region of the disk and we use 10 times more photons per wavelengths. The percentage of difference between this model and mod 0 in the IR regime amounts to less than $6 \%$. Deviations of about $10 \%$ in the optical range are due to differences in the number of photons.

To test the influence of the frequency resolution on our results we run the $2 \mathrm{MC}$ codes MC3D and RADMC doubling the number of walenghts where to solve the RT equation. The other three codes could not take part to the comparison because of not enough computer memory. To have reasonable runtime for MC3D (a couple of days), we restrict ourselves to the case $\tau_{\mathrm{v}}=10$ and we lower the number of photons in comparison to Table 3 (half in the case of RADMC and 4 times lower in the case of MC3D). The lower number of photons introduces larger
Table 4. Relevant models for the spatial resolution tests.

\begin{tabular}{llllll}
\hline \hline Model & $\# r$ & $\begin{array}{l}\Delta r \\
{[\mathrm{AU}]}\end{array}$ & $\# \theta$ & $\begin{array}{l}\Delta \theta \\
{\left[{ }^{\circ}\right]}\end{array}$ & $\begin{array}{l}\text { \#Phot } \\
{\left[\times 10^{6}\right]}\end{array}$ \\
\hline $\bmod 0$ & 55 & $0.03-141$ & 101 & 1.8 & 24.4 \\
$\bmod 1$ & 55 & $0.03-141$ & 31 & 5.8 & 24.4 \\
$\bmod 2$ & 40 & $0.3-141$ & 121 & 1.5 & 24.4 \\
$\bmod 3$ & 35 & $0.7-141$ & 121 & 1.5 & 24.4 \\
$\bmod 4$ & $10^{3}$ & $0.07-4.1$ & 121 & 1.5 & 244 \\
\hline
\end{tabular}

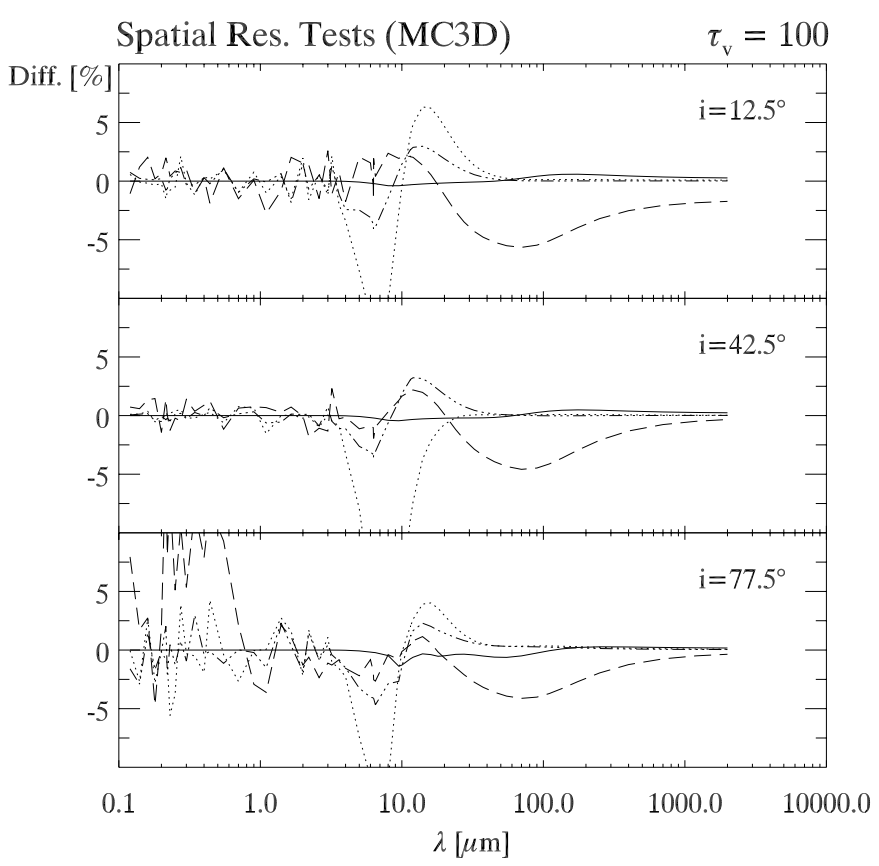

Fig. 9. Spatial resolution tests using the MC code MC3D. On the $y$-axis we plot the percentage of difference between the emerging SED of $\bmod 0$ and any other model in Table 4. Solid line: difference between mod 0 and mod1. Dot-dot dahsed line: difference between mod 0 and $\bmod 2$. Dotted line: difference between $\bmod 0$ and $\bmod 3$. Dashed line: difference between $\bmod 0$ and $\bmod 4$.

scatter at short wavelengths. Figure 10 shows the percentage of difference between MC3D and RADMC for the model with 61 wavelenghts (dotted line) and the new model with 122 wavelenghts (dashed line). Apart from the expected larger deviations at short wavelengths, the agreement between the two codes improves not more than $2 \%$ around $10 \mu \mathrm{m}$. Thus, we conclude that the nature of the IR deviations plotted in Fig. 8, is not due to coarse frequency sampling but to the different grid resolutions adopted by the codes (especially in radial direction) together with cumulative numerical errors.

\section{Discussion and conclusions}

Before presenting our findings, we briefly discuss the general features of the computed SEDs for different viewing angles ( $i$ ) and optical depths $(\tau)$. As mentioned in Sect. 2.2, optical depths are measured in the disk midplane from the inner to the outer boundary. Thus, the optical depths we refer to are the highest in the disk. 


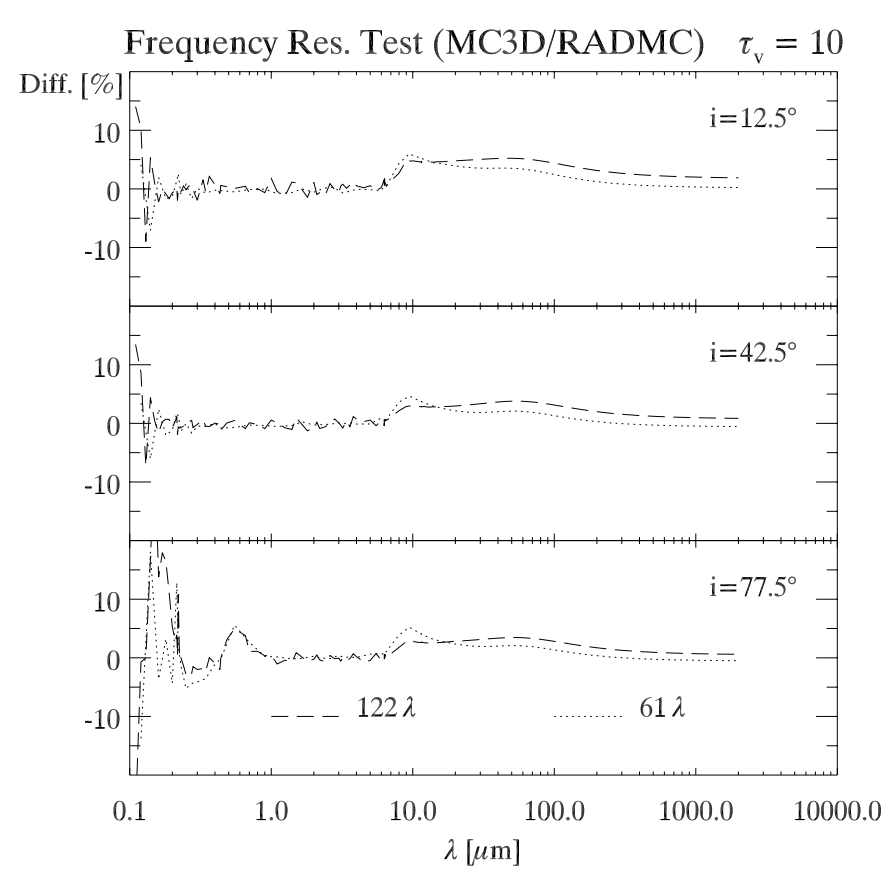

Fig. 10. Frequency resolution test using the MC codes MC3D and RADMC. On the $y$-axis we plot the percentage of difference between the emerging SED of the codes for two models, one sampling the frequency space with 61 (dotted line) and the other with 122 (dashed line) logarithmically distributed points.

Both panels of Fig. 7 show clearly that the far-infrared region (longward $100 \mu \mathrm{m}$ ) remains unaffected when varying the viewing angle. On the other hand, the short-wavelength part of the spectrum is strongly modified. When the disk is seen faceon the spectrum is dominated by unattenuated stellar radiation. As the disk inclination increases, more and more of the stellar flux is extincted by the dust in the disk. For the $\tau_{\mathrm{v}}=100$ case at $i=77.5^{\circ}$ this reduction amounts to a factor of $e^{-100}$ in the visual. However, due to the high albedo of the dust grains, a large fraction of the stellar radiation is scattered above the disk into the line of sight. Dust scattering is also responsible for the excess of emission at stellar wavelengths seen at small disk inclinations (see left panel of Fig. 7 especially for high optical depths). The optical depth also affects the strong $10 \mu \mathrm{m}$ feature produced by the $\mathrm{SI}-\mathrm{O}$ stretching. While in most cases the feature appears strongly in emission, for the $\tau_{\mathrm{v}}=100$ test case at $i=77.5^{\circ}$ the feature appears in absorption (see Fig. 7 right panel). The $20 \mu \mathrm{m}$ feature is much weaker than the $10 \mu \mathrm{m}$, but it is already visible for the model with $\tau_{\mathrm{v}}=1$. All these features are in agreement with earlier radiative transfer computations of disks (e.g. Efstathiou \& Rowan-Robinson 1990; Menshchikov \& Henning 1997).

Our aim in this paper is to provide benchmark solutions for the $2 \mathrm{D}$ continuum radiative transfer problem in circumstellar disks. The problems we present have optical depths up to 100, which is actually the limit of current computational capabilities for most of the codes. The corresponding total mass in the disk of about 0.01 solar masses covers most of the observed disks around low mass stars. For more massive disks around intermediate and high mass stars as well as tori obscuring active galactic nuclei, the numerical strategies have to be modified, using e.g. the diffusion approximation for high optical depths. We used five independent radiative transfer codes that implement different numerical schemes. We compare both the resulting temperature structure and the emerging SEDs. For the lowest optical depth case $\left(\tau_{\mathrm{v}}=0.1\right)$ we also compared the results against a semi-analytic solution which treat scattering only as extinction term. The other three cases $\left(\tau_{\mathrm{v}}=1,10,100\right)$ cannot be solved in a semi-analytic way, since multiple scattering and absorption-reemission events strongly affect the solution.

We find that the overall shape of the temperature distribution and of the emerging SEDs is well reproduced by all the codes. Differences in the temperature are smaller than $1 \%$ for all the codes in the most optically thin case. Even for the most optically thick model, differences in the temperature remain below $15 \%$. As for the SEDs, deviations among the codes are smaller than $3 \%$ at all wavelengths and disk inclinations for the most optically thin model. For the models with $\tau_{\mathrm{v}}=1$ and 10 at all disk inclinations and for the most optically thick case for disk inclinations of 12.5 and $42.5^{\circ}$, differences do not exceed $10 \%$. Only for the most optically thick case and an almost edge-on disk differences around $10 \mu \mathrm{m}$ exceed $20 \%$ in the case of STEINRAY. We stress that this is the case for which the numerics is the most difficult: the codes have to treat both a very optically thin atmosphere and a thick disk midplane. Independent tests using two of the MC codes show that the frequency resolution cannot account for the infrared deviations among the codes in the almost edge-on disk and the most optically thick model. Grid resolution especially in radial direction together with cumulative numerical errors play a major rule. The presented results provide a robust way to test other continuum RT codes and demonstrate the possibilities of the current computational capabilities. Temperature distributions and SEDs for all the test cases are available at the web site: http://www.mpia.de/PSF/PSFpages/RT/ benchmark.html

Acknowledgements. The developements of MCTRANSF has taken advantages of several discussions with Anne Dutrey to adapt its performances in agreement with the problems related to the disk of YSOs. C. P. Dullemond acknowledges support from the European Commission under TMR grant ERBFMRX-CT98-0195 ("Accretion onto black holes, compact objects and prototars").

\section{References}

Bachiller, R. 1996, ARA\&A, 34, 111

Bjorkman, J. E., \& Wood, K. 2001, ApJ, 554, 615

Cashwell, E. D., \& Everett, C. J. 1959, A practical manual on the Monte Carlo Method for random walk problems (New York: Pergamon)

Chiang, E. I., \& Goldreich, P. 1997, ApJ, 490, 368

Chiang, E. I., \& Goldreich, P. 1999, ApJ, 519, 279

Chini, R., Krügel, E., \& Kreysa, E. 1986, A\&A, 167, 315

Collison, A. J., \& Fix, J. D. 1991, ApJ, 368, 545

Cotera, A. S., Whitney, B. A., Young, E., et al. 2001, ApJ, 556, 958

Draine, B. T., \& Lee, H. M. 1984, ApJ, 285, 89

Dullemond, C. P., \& Turolla, R. 2000, A\&A, 360, 1187

Dullemond, C. P. 2003, A\&A, submitted

Dullemond, C. P., van Zadelhoff, G. J., \& Natta, A. 2002, A\&A, 389, 464 
Efstathiou, A., \& Rowan-Robinson, M. 1990, MNRAS, 245, 275

Efstathiou, A., \& Rowan-Robinson, M. 1991, MNRAS, 252, 528

Evans, A. 1994, The Dusty Universe (Chichester, New York: J. Wile), 67

Groenewegen, M. A. T. 1993, A\&A, 271, 180

Henning, Th. 1985, Astr. Space Sci., 114, 401

Henning, Th. 2001, The Formation of Binary Stars, IAU Symp., 200, 567

Hummer, D. G., \& Rybicki, G. B. 1971, MNRAS, 152, 1

Ivezic, Z., Groenewegen, M. A. T., Men'shchikov, A., \& Szczerba, R. 1997, MNRAS, 291, 121

Kikuchi, N., Nakamoto, T., \& Ogochi, K. 2002, PASJ, 54, 589

Kunasz, P. B., \& Auer, L. H. 1988, JQSRT, 39, 67

Lefèvre, J., Bergeat, J., \& Daniel, J. Y. 1982, A\&A, 114, 341

Lenzen, R. 1987, A\&A, 173, 124

Leung, C. M. 1976, ApJ, 209, 75

Lopez, B., Mékarnia, D., \& Lefèvre, J. 1995, A\&A, 296, 752

Lopez, B., \& Perrin, J.-M. 2000, A\&A, 354, 657

Lucy, L. B. 1999, A\&A, 344, 282

Malbet, F., \& Bertout, C. 1991, ApJ, 383, 814

Martin, P. G., Rogers, C., \& Rybicki, G. B. 1984, ApJ, 284, 317

McCaughrean, M. J., \& O’Dell, C. R. 1996, AJ, 111, 1977

Men'shchikov, A. B., \& Henning, T. 1997, A\&A, 318, 879

Mihalas, D. M., Auer, L. H., \& Mihalas, B. R. 1978, ApJ, 220, 1001

Mihalas, D., \& Mihalas, B. 1984, Foundations of radiation hydrodynamics (Oxford University Press)

Natta, A., Meyer, M. R., \& Beckwith, S. V. W. 2000, ApJ, 534, 838

Niccolini, G., Woitke, P., \& Lopez, B. 2003, A\&A, 399, 703
Rogers, C., \& Martin, P. G. 1984, ApJ, 284, 327

Rowan-Robinson, M. 1980, ApJS, 44, 403

Rybicki, G. B., \& Hummer, D. G. 1991, A\&A, 245, 171

Scoville, N. Z., \& Kwan, J. 1976, ApJ, 206, 718

Steinacker, J., Thamm, E., \& Maier, U. 1996, JQSRT, 56, 1, 97

Steinacker, J., Hackert, R., Steinacker, A., \& Bacmann, A. 2002a, JQSRT, 73, 557

Steinacker, J., Michel, B., \& Bacmann, A. 2002b, JQSRT, 74, 183

Steinacker, J., Bacmann, A., \& Henning, Th. 2002c, JQSRT, 75, 765

Steinacker, J., Henning, Th., Bacmann, A., \& Semenov, D. 2003, A\&A, 401, 405

Stone, J., Mihalas, D., \& Norman, M. 1992, ApJS, 80, 819

van Zadelhoff, G.-J., Dullemond, C. P., van der Tak, F. F. S., et al. 2002, A\&A, 395, 373

Winters, J. M., Dominik, C., \& Sedlmayr, E. 1994, A\&A, 288, 255

Wolf, S., Fischer, O., \& Pfau, W. 1998, A\&A, 340, 103

Wolf, S., \& Henning, Th. 1999a, A\&A, 341, 675

Wolf, S., Henning, Th., \& Stecklum, B. 1999b, A\&A, 349, 839

Wolf, S., \& Henning, Th. 2000, Comp. Phys. Comm., 132, 166

Wolf, S., Stecklum, B., \& Henning, Th. 2001a, in The Formation of Binary Stars, IAU Symp., 200, 295

Wolf, S. 2001b, A\&A, 379, 690

Wolf, S., Voshchinnikov, N. V., \& Henning, Th. 2002, A\&A, 385, 365

Wood, K., Wolff, M. J., Bjorkman, J. E., \& Whitney, B. 2002, ApJ, 564,887

Yorke, H. W., \& Shustov, B. M. 1981, A\&A, 98, 125

Yorke, H. W. 1985, Birth and infancy of stars (A86-31601 13-90). Amsterdam, North-Holland, 645 